\title{
Murciélagos (Mammalia: Chiroptera) del área urbana del municipio de Boa Vista, Roraima, Brasil
}

\author{
Ubirajara Dutra Capaverde Junior ${ }^{1 *}$, Susi Missel Pacheco $^{2} \&$ Marcos Eugenio Duarte $^{3}$ \\ ${ }^{1}$ Instituto de Nacional de Pesquisas Amazônicas, Av. André Araújo, 2936 Aleixo, Caixa Postal: 2223 \\ CEP: 69060-001 Manaus, Amazonas, Brasil. \\ ${ }^{2}$ Instituto SAUVER, Av. Pernambuco, 2623, sala 404, Bairro Floresta, CEP 90240-005, Porto Alegre, RS, Brasil. \\ ${ }^{3}$ Agencia de Defesa Agropecuária de Roraima, Rua Coronel Mota, nº 1142, Centro \\ CEP: 69.305-120, Boa Vista, Roraima, Brasil. \\ *Corresponding author e-mail: capaverdejunior@hotmail.com
}

DOI: http://dx.doi.org/10.14709/BarbJ.7.1.2014.03

English title: Species of bats (Chiroptera) in urban area of the Boa Vista, Roraima

\begin{abstract}
Resumen
La mayoría de los trabajos con murciélagos en la Amazonía brasileña ha sido realizada en ambientes forestales. Pocos estudios se centran en áreas de sabana amazónica y son rarísimos en las áreas urbanas, lo que resulta en una submuestra de incidencia de esos mamíferos. Esta investigación ha proporcionado la lista de quirópteros que viven en el área urbana de Boa Vista-Roraima y ha contribucido al aumento de datos de distribución y ocurrencia en Brasil. Se trata de una investigación no experimental del tipo exploratorio y descriptivo, con enfoque cuantitativo. Como resultados se han identificado 23 especies en el área urbana, distribuidas en cinco familias y con dieta predominantemente frugívora. El número de especies es similar al que viene registrándose en los inventarios de otras capitales en Brasil.
\end{abstract}

Palabras clave: Inventario; Quirópteros; Región Amazónica

\begin{abstract}
While most work on bat research in the Brazilian Amazon has been held in forests, few studies were placed in Amazonian savannas and even less in urban areas, resulting in a subsampling of these mammals distribution. The study surveyed the bats that inhabit the urban area of Boa Vista, Roraima and contributed to the data growth occurring in Brazil. It is a non-experimental research but exploratory and descriptive, with quantitative focus. We identified 23 species in the urban area, distributed in five families and showing predominantly frugivorous diet. This figure is similar to the number of species that have been recorded in inventories from other Brazilian capitals.
\end{abstract}

Key words: Amazon Region; Chiroptera; Inventory, Check-list 


\section{INTRODUCCIÓN}

Actualmente el proceso de transformación del paisaje natural en grandes centros urbanos trae como consecuencia la disminución de la biodiversidad local y, a veces, regional, aunquesegún Yalden (1980), los pequeños mamíferos, entre ellos los quirópteros, podrían sobrevivir en ambientes antropizados. Sin embargo, Pedro (1998) afirmó que solamente el $20 \%$ de las especies murciélagos en Brasil serían capaces de mantener poblaciones estables en este ambiente.

Los murciélagos que ocurren en áreas urbanas a menudo presentan mayor habilidad en la búsqueda y en el uso del alimento, agua y de refugios disponibles que les permite mantenerse en este ambiente antropizado y teóricamente hostil (Pacheco et al., 2008).

La mayoría de los murciélagos presentes en ambientes urbanos son insectívoros, y en ambientes urbanos la iluminación pública y de las casas suele atraer insectos. Además hay cierta similitud entre los abrigos naturales de estas especies disponibles en ambiente urbano (Aguita, 2003; Pacheco et al., 2008). Con relación a las especies nectarívoras y frugívoras usualmente detectadas en este ambiente, buscan abrigo y alimentos junto a zonas verdes, en vías públicas, plazas, parques y áreas particulares (Uieda et al., 2008).

En Brasil hay 174 especies de quirópteros registradas pertenecientes a nueve familias (Paglia et al., 2012), de las cuales 63 se ha provado que utilizan el ambiente urbano en un mínimo de 18 ciudades brasileñas (Lima, 2008), siendo Manaus la única ciudad citada para la región Norte con 24 especies. Pacheco et al. (2010) registraron 47 especies para áreas urbanas en seis estados brasileños São Paulo (SP), Santa Catarina (SC), Rio Grande do Sul (RS), Rio de Janeiro (RJ), Paraná (PR), Mato Grosso (MT), además del Distrito Federal (DF). En estas regiones predominaron las especies de la familia Phyllostomidae (17), seguidas de las familias Molossidae (16), Vespertilionidae (11), Emballonuridae (2) y Noctilionidae (1), de las cuales 30 especies son insectívoras, 13 nectarívoras/frugívoras, dos omnívoras, una hematófaga y una piscívora.

Tanto en Brasil como en el resto del mundo, todavía existe poca información respecto a la conservación y ecología de estos mamíferos en áreas urbanas y naturales. El objetivo de ese estudio fue básicamente identificar las especies de quirópteros que ocurren en el ambiente urbano de Boa Vista, Roraima y proporcionar una lista de especies actualizada y completa.

\section{Área de estudio}

De acuerdo con SEBRAE (2006), el municipio de Boa Vista comprende un área de $5.711,9 \mathrm{~km}^{2}$, abrigando la capital del Estado de Roraima, y ubicada en la región central del Estado, entre las coordinadas $02^{\circ} 49^{\prime} 11^{\prime \prime} \mathrm{N}$ y $60^{\circ} 40^{\prime} 24^{\prime \prime} \mathrm{W}$ (Figura 1), a una altitud de $90 \mathrm{~m}$ sobre el nivel del mar. Presenta clima, según Köppen (1948), tropical húmedo del tipo "A", del subtipo AW: clima tropical lluvioso, , es caluroso y húmedo, con estación lluviosa en el verano con predominio de sabanas.
El estudio se ciñó al área urbana del referido municipio delimitada por las coordinadas geográficas: $2^{\circ} 53^{\prime} 54.53^{\prime \prime}$, $2^{\circ} 44^{\prime} 18.95^{\prime \prime}, 2^{\circ} 51^{\prime} 23.28^{\prime \prime}, 2^{\circ} 48^{\prime} 42.31$ ”N; y $60^{\circ} 41^{\prime} 7.64^{\prime \prime}$, $60^{\circ} 43^{\prime} 19.15^{\prime}, 60^{\circ} 37^{\prime} 36.57^{\prime \prime}, 60^{\circ} 47^{\prime} 31.85^{\prime \prime}$ 'W. A partir de esos puntos se delineó un polígono imaginario que restringió el área estudiada a $154 \mathrm{~km}^{2}$ de área efectivamente urbana, y configurada en zonas: leste, oeste, norte y sur (Figura 2).

\section{Material y Métodos}

Se realizaron veintitrés noches de muestreo con dos redes de niebla, ejecutando un esfuerzo de muestra de $4997.7 \mathrm{~h} /$ $\mathrm{m}^{2}$ según el método de Straube y Bianconi (2002), y siete búsquedas activas de tres horas cada una en construcciones (tejados, paredes de ladrillos, huecos entre edificios), dando un total de $21 \mathrm{~h}$ (Tabla 1). Las colectas se realizaron teniendo en cuenta las fases de la luna, intentando realizar el máximo número de noches durante la fase menguante o nueva.

Las redes de niebla fueron dispuestas al nivel del suelo hasta $3 \mathrm{~m}$ de altura, próximas a plantas en fructificación, floración, refugios y/o en posibles rutas de vuelo. Los murciélagos se extrayeron de la red inmediatamente después de la captura y fueron mantenidos en sacos de captura. Este método basadoen la baja altura de las redes y al ambiente rico en plantas en fructificación probablemente haya propiciado una mayor captura de especies de la familia Phyllostomidae, en relación a otras especies insectívoras.

Las construcciones humanas se evaluaron esporádicamente en los sitios de muestras descriptos en la Tabla 1, con el objetivo de identificar las especies que usualmente no son capturadas en redes de niebla. Para colectar los murciélagos en estos locales se utilizaron pinzas de $30 \mathrm{~cm}$ o redes de mano unidas aun cable extensible de hasta tres metros de largo.

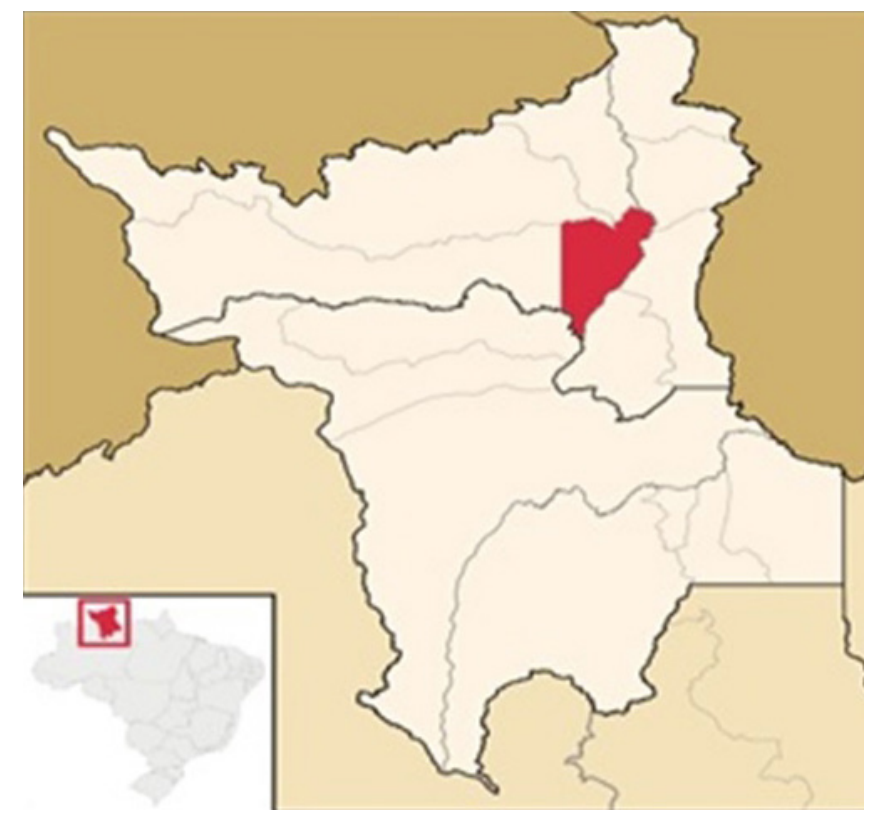

Fig. 1 - Mapa físico del Estado de Roraima, localización en Brasil (recuadro pequeño) y localización del municipio de Boa Vista (en rojo). Fuente: SEBRAE (2006). 


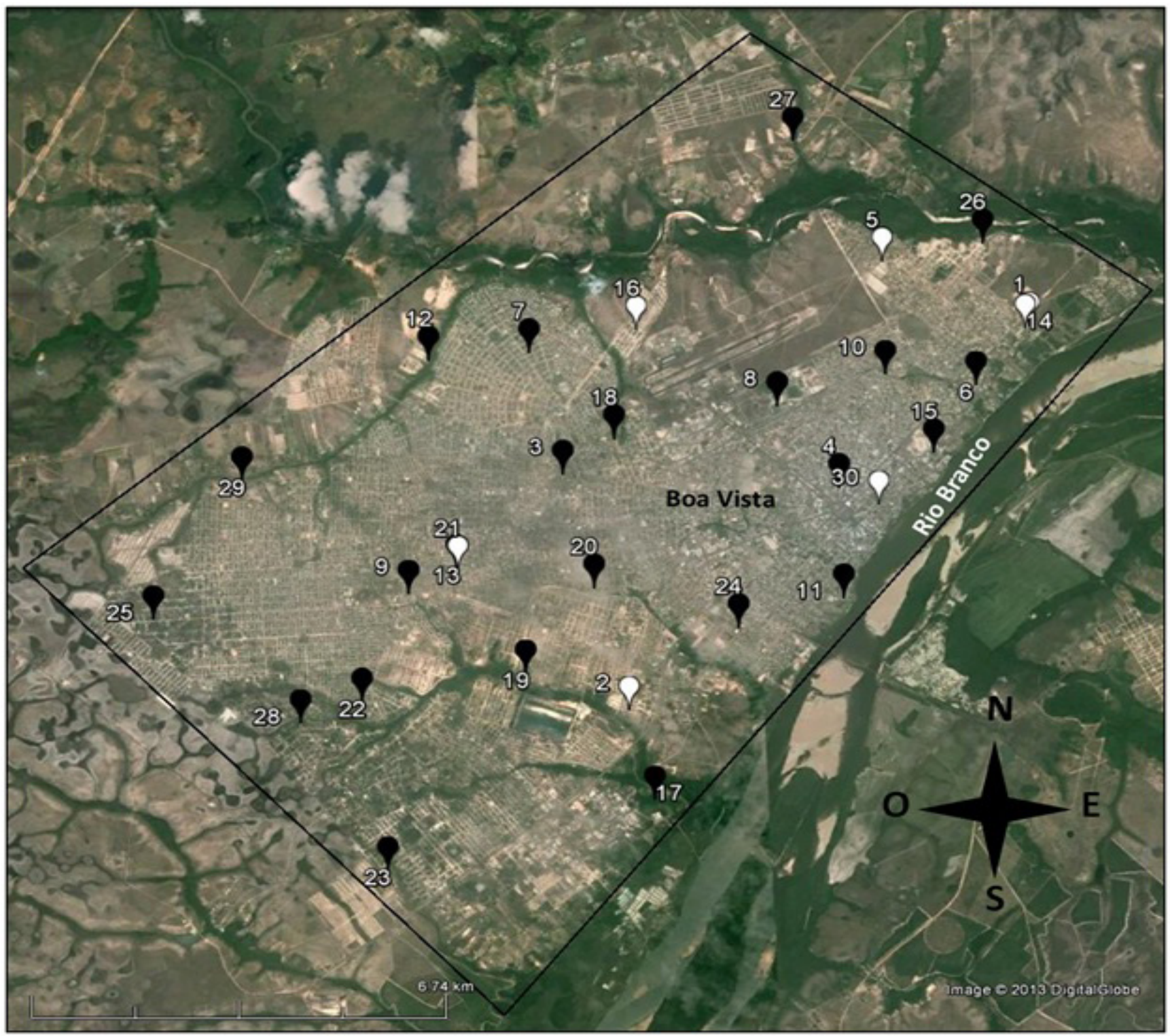

Fig. 2 - Ciudad de Boa Vista, los puntos negros representan las colectas con auxilio de redes, los puntos blancos, las colectas en refugios y el polígono negro, el límite del área muestreada. Fuente: Google Earth (2013).

Las identificaciones taxonómicas se basaron en caracteres mofológicos siguiendo a Vizotto y Taddei (1973), Barquez et al. (1993), Gregorin y Taddei (2002) y Aguirre (2009).

Un ejemplar de cada especie fue colectado como material testigo para una identificación específica. Los murciélagos colectados fueroneutanizados y depositados en el Instituto Sauver como material de referencia, en conformidad con la licencia 30045-1/SISBIO. El procedimiento de eutanasia $\mathrm{y}$ anestesia fue mediante Isoflurano $1 \mathrm{ml} / \mathrm{ml}$ (Nombre Comercial: "FORANE") y posterior acetato de etilo, conforme prescrito por el veterinario Dr. Marcos Eugenio Duarte, responsable técnico de esta investigación. No se cita referencia bibliográfica, debido a la falta de descripción específica para pequeños mamíferos. Se realizaron medidas corporales y cranianas, y fueron verificadas las masas corporales e identificadas las fórmulas dentarias. Tras eso, los especímenes fueron etiquetados y mantenidos congelados en el laboratorio de Zoología de la Facultad Cathedral hasta su envío al Instituto Sauver, donde han sido taxidermizados o conservados en alcohol (70\%) e inventariados en la Colección de Referencia de la institución (IS de 0100-00123). Los especímenes no inventariados fueron liberados, después de su evaluación morfométrica y dentaria, en los mismos puntos de captura durante la misma noche de capturas.

\section{Resultados Y Discusión}

Se ha capturado un total de 602 murciélagos (23 colectados), pertenecientes a cinco familias y 23 especies para el área urbana de Boa Vista (Tabla 1), representando el $13 \%$ de la riqueza de murciélagos sugerida para Brasil ( $\mathrm{n}=$ 174) y el $16 \%$ de la riqueza de murciélagos sugerida para la Amazonía ( $\mathrm{n}=146)$, según Paglia et al. (2012).

Comparando las 23 especies identificadas en Boa Vista con el estudio de Lima (2008), se verifica que las especies Rhynchonycteris naso, Saccopteryx bilineata, Micronycteris minuta, Lophostoma silvicolum, Trinycteris nicefori y Ametrida centurio no estaban registradas para el área urbana de otras ciudades brasileñas. La familia mejor representada fue Phyllostomidae con 353 individuos capturados y 12 especies, seguida de Molossidae 176 individuos y 4 especies, 
Tabla 1 - Lista de familias y especies por gremio alimenticio de quirópteros registradas para el área urbana, en las cuatro zonas de muestra en Boa Vista, de agosto de 2011 a julio de 2012.

\begin{tabular}{|c|c|c|c|c|c|c|}
\hline \multirow{2}{*}{ Especie } & \multirow{2}{*}{ Nicho trófico } & \multicolumn{4}{|c|}{ Número de individuos } & \multirow{2}{*}{ Tota } \\
\hline & & Norte & Sur & Este & Oeste & \\
\hline Emballonuridae & & 1 & & 16 & 1 & 18 \\
\hline Rhynchonycteris naso (Wied-Neuwied, 1820) & Insectívoro & & & 16 & & 16 \\
\hline Saccopteryx bilineata (Temminck, 1838) & Insectívoro & 1 & & & 1 & 2 \\
\hline Noctilionidae & & $\mathbf{0}$ & 50 & $\mathbf{0}$ & $\mathbf{0}$ & 50 \\
\hline Noctilio albiventris Desmarest, 1818 & Insectívoro & & 50 & & & 50 \\
\hline Phyllostomidae & & 17 & 103 & 15 & 218 & 353 \\
\hline Micronycteris minuta (Gervais, 1856) & Insectívoro & & & 2 & & 2 \\
\hline Phyllostomus discolor Wagner, 1843 & Omnívoro & & 5 & & 7 & 12 \\
\hline Lophostoma silvicolum d'Orbigny, 1836 & Insectívoro & & & & 1 & 1 \\
\hline Trinycteris nicefori (Sanborn, 1949) & Insectívoro & & & 1 & & 1 \\
\hline Glossophaga soricina (Pallas, 1766) & Polinívoro & & 2 & 3 & 4 & 9 \\
\hline Carollia perspicillata (Linnaeus, 1758) & Frugífero & 4 & 9 & 1 & 5 & 19 \\
\hline Ametrida centurio Gray, 1847 & Frugífero & & & & 1 & 1 \\
\hline Artibeus cinereus (Gervais, 1856) & Frugífero & & & & 1 & 1 \\
\hline Artibeus lituratus (Olfers, 1818) & Frugífero & 3 & 22 & 2 & 7 & 34 \\
\hline Artibeus planirostris (Leach, 1821) & Frugífero & 10 & 65 & 5 & 186 & 266 \\
\hline Artibeus obscurus (Schinz, 1821) & Frugífero & & & 1 & 5 & 6 \\
\hline Platyrrhinus lineatus (Geoffroy, 1810) & Frugífero & & & & 1 & 1 \\
\hline Vespertilionidae & & & 1 & & 4 & 5 \\
\hline Eptesicus brasiliensis (Desmarest, 1819) & Insectívoro & & 1 & & & 1 \\
\hline Eptesicus furinalis (d'Orbigny, 1847) & Insectívoro & & & & 2 & 2 \\
\hline Lasiurus egregius Peters, 1870 & Insectívoro & & & & 1 & 1 \\
\hline Myotis albescens (Geoffroy, 1806) & Insectívoro & & & & 1 & 1 \\
\hline Molossidae & & 1 & 71 & 22 & 82 & 176 \\
\hline Cynomops planirostris Peters, 1865 & Insectívoro & & 20 & & & 20 \\
\hline Molossops temminckii Burmeister, 1854 & Insectívoro & 1 & & & 2 & 3 \\
\hline Molossus molossus (Pallas, 1766) & Insectívoro & & 50 & 22 & 80 & 152 \\
\hline Promops nasutus (Spix, 1823) & Insectívoro & & 1 & & & 1 \\
\hline Total & & 19 & 225 & 53 & 305 & 602 \\
\hline
\end{tabular}

Vespertilionidae 5 individuos y 4 especies, Emballonuridae 18 individuos y 2 especies y Noctilionidae 50 individuos y 1 especie (Tabla 1). De acuerdo con Kunz y Kurta (1988), eso puede ser debido a la metodología, concretamente por el uso de redes de niebla que favorece la captura de filostómidos mientras que la búsqueda de refugios en construcciones favorece la captura de molósidos y vespertiliónidos. En el caso de $N$. albiventris el número de individuos elevado se debe a la captura realizada en la proximidad de la salida del abrigo.

El predominio de la familia Phyllostomidae en el municipio de Boa Vista, confirma los resultados obtenidos en ambientes urbanos por Pacheco et al. (2010) para seis estados de Brasil, que revelan el predominio de la familia Phyllostomidae en las capturas con redes. Sin embargo, el número de especies estrictamente frugívoras (8) es menor que el de las especies insectívoras (12). Se verifica en la
Tabla 1 donde solo la especie $A$. planirostris, es responsable para la abundancia de la familia Phyllostomidae en relación a las otras, ocurriendo lo mismo para M. molossus y la familia Molossidae.

Las familias Vespertilionidae, Emballonuridae y Molossidae presentaron tasas de captura más bajas que la familia Phyllostomidae. Este se puede deber al diferente esfuerzo de captura en refugios; muchos lugares muestreados o bien no tenían murciélagos ni vestigios de poblaciones, o bien cuando estaban en tejados, eran de difícil captura y observación, es decir, a demasiada altura (superior a $3 \mathrm{~m}$ ) o bajos (inferior a $50 \mathrm{~cm}$ de altura).

Aún presentando diferente esfuerzo de captura en las cuatro zonas de la ciudad de Boa Vista, se prueba que Artibeus lituratus y $A$. planirostris están presentes en las cuatro zonas urbanas (Tabla 1), así como Molossus molossus, 
con un mayor número de capturas mientras que Saccopteryx bilineata, Lophostoma silvicolum, Trinycteris nicefori, Ametrida centurio, Artibeus cinereus, Platyrrhinus lineatus, Eptesicus brasiliensis, Lasiurus egregius, Myotis albescens y Promops nasutus se capturaron en una única ocasión para el área urbana de Boa Vista.

Los primeros estudios realizados en el estado de Roraima se llevaron a cabo en áreas protegidas (Taddei y Reis, 1980; Mok et al., 1982; Robinson, 1998). Recientemente Souza (2011) realizó un último estudio en área urbana, donde registró para el Estado 18 especies y 11 para Boa Vista. En el presente estudio, han sido capturadas 23 especies, incluyendo 12 nuevas especies para la localidad.

Es preocupante la actual expansión urbana de la ciudad de Boa Vista y se hace necesario conocer y comprender mejor la ecología y el comportamiento de las especies mostradas. A diferencia de otras áreas urbanas del país, la quiropterofauna de Boa Vista aún usa principalmente el ambiente periurbano con refugios próximos a la vegetación, provocando que pocas especies y pocos individuos se encuentren en construcciones. Los estudios se deberían complementar con la realización de una educación ambiental más enfocada a la conservación de los murciélagos, que contribuya a la desmitificación de estos, y que al contrario presente a la comunidad su importancia económica y ecológica.

\section{Agradecimientos}

Agradecemos a la Facultad Cathedral, al Prof. Alexandre Castilho, a Ubirajara Dutra Capaverde Neto, a Maria Fernanda Bessa, a Dr ${ }^{\text {a }}$ Solange Capaverde, al Prof. Dr. Paulo Sergio Ribeiro de Mattos y a Med. Vet. Sra Ana Nóbrega.

\section{REFERENCIAS}

Aguirre, L.F.; Vargas, A.; Solari, S. 2009. Clave de campo para la identificación de los murciélagos de Bolivia. Centro de Estudios en Biología Teórica y Aplicada. Cochabamba: Etreus. 38 p.

Aguita, R. 2003. Urbanitas con patas: animales en la gran ciudad. Ambienta, Madrid, v. 20, pp.32-37.

Barquez; M. R.; Giannini, P. N.; Mares, A. M. 1993. Guide to the bats of Argentina. Oklahoma: Museum of Natural History, 282 p.

Gregorin, R.; TAddeI, V. A. 2002. Chave artificial para a identificação de molossídeos brasileiros (Mammalia, Chiroptera). Mastozoología Neotropical, Tucumán, v. 9, n. 1, pp. 13-32.

Köppen, W. 1948. Climatologia: con un estudio de los climas de la tierra. Fondo de Cultura Econômica. México. 479 p.

KunZ, T. H.; KurTA, A. 1988. Capture methods and holding devices. pp. 1-29. In: (KunZ, T. H. 1998. (Ed). Ecological and behavioral methods for the study of bats. Washington: Smithsonian Press.)
LimA, I. P. 2008. Espécies de morcegos (Mammalia, Chiroptera) registradas em parques nas áreas urbanas do Brasil e suas implicações no uso deste ambiente. pp. 71-85. In: (Reis, N. R.; Peracchi, A. L.; SANtos, G. A. S. D. (Ed.). Ecologia de morcegos. Londrina: Technical Books.)

Mok, W. Y.; Wilson, D. E.; Lacey, L. A.; Luizão, R. C. C. 1982. Lista atualizada de quirópteros da Amazônia brasileira. Acta Amazônica, Manaus, v. 12, pp. 81723.

Pacheco, S. M.; Marques, R. V.; Grillo, H.; Marder, E.; Bianconi, G.; Miretzki, M.; Passos I. L.; Rosa, V. A. 2008. Morcegos urbanos da Região Sul do Brasil. pp. 415-426. In: (Pacheco, S. M.; Marques, R. V.; EsbÉRARD, C. E. L. (Org.). Morcegos no Brasil: biologia, sistemática, ecologia e conservação. Porto Alegre: A. Digital.)

Pacheco, S. M.; Sodré, M.; Gama, A. R.; Bredt, A.; Cavallini, E. M.; Marques, R. V.; Guimarães, M. M.; BianCONI, G. 2010. Morcegos urbanos: status do conhecimento e plano de ação para a conservação no Brasil. Chiroptera Neotropical, Brasília, v. 16, n. 1, pp. 629-647.

Paglia, A. P.; Fonseca, G. A. B.; Rylands, A. B.; Herrmann, G.; Aguiar, L. M. S.; Chiarello, A. G.; Leite, Y. L. R.; Costa, L. P.; Siciliano, S.; KierulfF, M. C. M.; Mendes, S. L.; Tavares, V. C.; Mittermeier, R. A.; PATTON, J. L. 2012. Lista anotada dos mamíferos do Brasil. 2 ed. Occasional Papers in Conservation Biology, Arlington, n. 6, pp. 1-76.

Pedro, W. A. 1998. Diversidade de morcegos em habitats florestais fragmentados do Brasil. 1998. $128 \mathrm{f}$. Tese (Doutorado em Ecologia e Recursos Naturais) Universidade Federal de São Carlos, São Carlos.

Robinson, F. 1998. The bats of the Ilha de Maracá. In: J.A. Ratter, J.A.; Milliken, W. (Eds.). Maracá: the biodiversity and environment of an Amazonian Rainforest. London: John Wiley \& Sons. pp. 165-188.

SEBRAE - SERVIÇO BRASILEIRO DE APOIO ÀS MICRO E PEQUENAS EMPRESAS. 2006. Série Diagnósticos Municipais: Município de Boa Vista. 3 ed. Boa Vista: Sebrae-RR, $94 \mathrm{p}$.

Souza, J.R. 2011. Pesquisa do vírus da raiva em quirópteros no estado de Roraima. 63 f. Dissertação (Mestrado em Recursos Naturais) - Universidade Federal de Roraima, Boa Vista.

Straube, F.C. \& Bianconi, G. 2002. Sobre a grandeza e a unidade utilizada para estimar esforço de captura com utilização de redes de niebla. Chiroptera Neotropical, Brasília, v. 8, n. 1/2, pp. 150-152.

TAdDEI, V. A.; ReIs, N. R. 1980. Notas sobre alguns morcegos da Ilha de Maracá, Território Federal Roraima (Mammalia, Chiroptera). Acta Amazônica, Manaus, v. 10, pp. 363-68. 
Uieda, W.; Bredt, A.; Pinto, P. P. 2008. Dieta, abrigos e comportamento fitófago de Artibeus lituratus (Phyllostomidae) em Brasília, DF, e sua relação com as plantas usadas na arborização urbana. pp. 427-443. In: (PACheco, S. M.; MARques, R. V.; EsbÉRARD, C. E. L. (Org.). Morcegos no Brasil: biologia, sistemática, ecologia e conservação. Porto Alegre: A Digital.)

Vizotto, L. D.; TAdDeı, V.A. 1973. Chave para determinação de quirópteros brasileiros. São José do Rio Preto: Francal. 72 p.

YALDEN, D. W. 1980. Notes on the diet of urban Kestrels. Bird Study, London, v. 27, pp. 235-238. DOI: https:// dx.doi.org/10.1080/00063658009476683 